\title{
Brain Stimulation Reward
}

\author{
Peter Shizgal
}

Groupe de recherche en neurobiologie comportementale and Department of Psychology

Concordia University

phone: +1 (514) 848-2424 ext 2191

fax: 1 (514) 848-2817

peter.shizgal@concordia.ca

http://csbn.concordia.ca/Faculty/Shizgal/ 


\section{Abstract}

In 1953, Olds and Milner discovered that rats would readily learn to work for electrical stimulation of certain brain sites. Their findings inspired a large body of research on the neural basis of reward, motivation, and learning. Unlike consummatory behaviors, which satiate as a result of ingestion of and contact with the goal object, performance for rewarding brain stimulation is remarkably stable and persistent. Pursuit of the stimulation is enhanced by different classes of dependence-inducing drugs, suggesting that common neural mechanisms underlie the rewarding effects of drugs and electrical brain stimulation. Indeed, dopaminecontaining neurons in the midbrain are implicated in both phenomena. Major schools of thought that have addressed brain stimulation reward differ with regards to the roles played by hedonic experience and craving, although there is substantial overlap between the different viewpoints. A tradition that arose in the study of machine learning has been brought to bear on the role of dopamine neurons in reward-related learning in animals and on the phenomenon of intracranial self-stimulation. Neuroeconomic perspectives strive to integrate the processing of benefits, costs, and risks into an account of decision making grounded in brain circuitry. Adjudication of the differences between the various viewpoints and progress towards identifying the relevant neural circuitry has been hindered by the lack of specificity inherent in the use of electrical stimulation to study central nervous system function. Many neurons in addition to those targeted are likely activated by such stimulation. Recently developed optogenetic methods may overcome this obstacle, providing much more specific means for stimulating or silencing populations of nerve cells selected on the basis of their gene expression, cell-body location, and projections. Coupled with behavioral methods of increasing sophistication and specificity and with quantitative modeling of signal flow in the relevant neural circuitry, the new optogenetic methods promise to bring us much closer to fulfillment of the hopes engendered long ago by the discovery of brain stimulation reward. 


\section{The Discovery and its Context}

Brain stimulation reward was discovered in 1953 by James Olds and Peter Milner (Olds \& Milner 1954; Milner 1989; Olds 1973). They had come to McGill University to work with D. O. Hebb, inspired by his groundbreaking theoretical work. In contrast to the dominant behaviorist orthodoxies of the day, which isolated psychology from the emerging disciplines of neuroscience and cognitive psychology, Hebb's views linked brain, mind, and behavior.

New findings and ideas about the neural bases of motivation and reinforcement provided a rich context for the discovery of BSR (Valenstein 1973). For example, the demonstration that lesions of different hypothalamic nuclei could lead either to massive overeating or starvation contributed to a seminal, physiologically based theory of motivation (Stellar 1954). During that era, English-speaking investigators became familiar with Hess' use of electrical stimulation to map the hypothalamic control of autonomic outflow and behavior (Hess 1949). Among Hess' observations were stimulation-elicited responses suggesting states of rage or fear. Shortly before Olds and Milner's discovery, Delgado, Roberts and Miller (Delgado et al. 1954) showed that cats would learn to escape electrical stimulation of certain deep forebrain structures. Another key component of the context was research linking brainstem circuitry to the control of arousal and vigilance (Moruzzi \& Magoun 1949). The pattern of connections between nerve cells in the brainstem region of interest was seen as net-like ("reticulated"); thus the region was dubbed the "reticular formation."

At the time that Olds joined Hebb's group, Milner and Seth Sharpless, another graduate student, had developed a theory linking reticular activation to positive reinforcement, the process by which desirable outcomes strengthen the behaviors that produce them. Their experimental tests of this idea proved disappointing: The rats tended to avoid locations in a maze where stimulation of the reticular formation had been delivered.

A strikingly different reaction was noted in a rat prepared by Olds. The electrode had somehow missed its reticular-formation target and ended up instead in the basal forebrain. This subject returned repeatedly to the location where stimulation had been delivered. As the experimenters altered the rewarded location, the animal's behavior changed accordingly, and it directed its searching towards the site where it had most recently hit pay dirt. Olds then succeeded in training the rat to press a lever that triggered delivery of the stimulation. This animal worked indefatigably to stimulate its own brain. Such behavior has since been termed "intracranial self-stimulation" (ICSS), and the effect that causes the animal to seek out the stimulation is called "brain stimulation reward" (BSR).

In the initial report published by Olds and Milner (Olds \& Milner 1954), they presciently discuss their findings "as possibly laying a methodological foundation for a physiological study of the mechanisms of reward." Indeed, their paper was the well-spring for thousands of subsequent experiments.

Why did the report by Olds and Milner have such a large and enduring impact? Perhaps paramount is the promise their results offered that psychological phenomena such as learning, reinforcement, and motivation could be investigated fruitfully by physiological means. The 
authors also pointed out that the phenomenon of BSR could be used to distinguish between competing behavioral theories. For example, a prominent theory at the time held that reinforcement arose from the reduction of a drive, such as the abatement of hunger. This view is not easily reconciled with the fact that rewarding stimulation of many sites arouses the animal rather than calming it and that stimulation of some BSR sites can also induce sated animals to engage in consummatory behaviors such as eating and drinking (Margules \& Olds 1962; Hoebel \& Teitelbaum 1962).

\section{The Phenomenon}

The behavior of the self-stimulating rat is striking to behold, particularly in the case of electrodes arrayed along the medial forebrain bundle (MFB). When the rewarding stimulation is strong and easily triggered, the rat attacks the lever with singular focus, pressing repeatedly and forcefully at high rates. This behavior is remarkably persistent. For example, a rat working for MFB stimulation maintained an average response rate above 2000 presses per hour over a continuous 26-hour period (Olds 1958a). Rats have been shown to cross an electrified grid (Olds $1958 b$ ) or to gallop uphill along a runway, leaping over hurdles (Edmonds \& Gallistel 1974) in order to access a lever that triggers delivery of the stimulation. When food was provided uniquely in an ICSS chamber in which strong stimulation was available, rats worked for the electrical reward in lieu of feeding and thus starved themselves (Routtenberg \& Lindy 1965).

Why would an animal work so assiduously for a reward that has no evident biological value? It has long been suspected that the neural activity triggered by the stimulation mimics some aspects of natural rewards. Indeed, MFB stimulation is as good an economic substitute for food and water as they are for themselves (Green \& Rachlin 1991), and the rewarding effect of such stimulation both competes and summates with the rewarding effects of sweet (Conover \& Shizgal 1994a) or salty (Conover et al. 1994) solutions. The incompleteness of the mimicry may give rise to some of the differences between BSR and natural rewards. Unlike performance for natural consummatory rewards, ICSS does not result in the accumulation of a substance in the body, which may explain why the appetite for stimulation does not satiate. Similarly, the electrical reward bypasses peripheral receptors for taste, smell, and touch, which may explain why the accumulation of nutrients in the gut undermines the rewarding effect of a gustatory stimulus (sucrose) more severely than the rewarding effect of MFB stimulation (Conover \& Shizgal 1994b).

The nature of the rewarding effect elicited by electrical stimulation may vary both across and within brain sites. For example, food or water deprivation can alter preference between stimulation of different brain sites (Gallistel \& Beagley 1971), and the potentiating effect of chronic food restriction is seen only at some MFB sites (Blundell \& Herberg 1968). Moreover, the fat-signaling hormone, leptin, has opposite effects on self-stimulation of different brain sites, depending on whether or not the rewarding effect is sensitive to chronic food restriction (Fulton et al. 2000). 


\section{Anatomy and Neurochemistry}

The sites where BSR can be produced occupy a substantial proportion of the brain volume (Wise 1996). Positive sites are distributed from the olfactory bulbs, at the front of the brain, to the nucleus of the solitary tract, at the back, and are found both in cortical and sub-cortical regions of the forebrain. The best-characterized sites lie along the trajectories of the medial forebrain bundle (MFB) and of fibers coursing near the mid-line of the brainstem. In both cases, the neurons that carry the reward-related signal away from the electrode tip include cells with relatively fast-conducting, longitudinally oriented, myelinated axons (Shizgal 1997; Miguelez \& Bielajew 2004). This characterization narrows the field of plausible candidates, but it leaves open multiple possibilities. The recent technical innovations described in the last section of this essay hold great promise for the long-sought identification of these neurons.

Several neurotransmitter systems are implicated in BSR. There is a large body of evidence tying dopamine-containing neurons to the rewarding effect (Wise \& Rompré 1989). ICSS is potentiated by manipulations that enhance transmission in these cells and weakened by manipulations that decrease dopaminergic neurotransmission. Recently, Witten, Steinberg and their co-workers demonstrated that rats will work for direct optical activation of midbrain dopamine neurons (Witten et al. 2011). The dopamine-containing neurons that project from the ventral tegmental area of the midbrain to the nucleus accumbens, a structure at the base of the anterior forebrain, are thought to play a particularly important role in BSR as well as in the effects of natural rewards such as food and water. Mice have been shown to self-stimulate for direct optical activation of excitatory inputs to the nucleus accumbens from glutamate-containing neurons in other forebrain structures (Stuber et al. 2011; Britt et al. 2012).

Although ventral tegmental dopamine neurons play a crucial role in BSR and project to forebrain structures via the MFB, their axons are difficult to excite under the conditions of ICSS experiments. Instead, or in addition, the rewarding effect may well arise from activation of inputs to the ventral tegmental dopamine neurons (Shizgal \& Murray 1989; Shizgal 1997; Moisan \& Rompré 1998).

Acetylcholine-containing neurons in the pedunculopontine and laterodorsal tegmental nuclei play a role in BSR, are activated transsynaptically by rewarding MFB stimulation (Yeomans et al. 2000; Rada et al. 2000), and provide excitatory input to ventral tegmental dopamine neurons (Forster et al. 2002). Mice will work to disinhibit dopamine neurons in the ventral tegmental area via a disynaptic GABAergic pathway arising in the bed nucleus of the stria terminalis in the basal forebrain (Jennings et al. 2013), and there are multiple pathways employing glutamate as a neurotransmitter that provide excitatory input to the ventral tegmental area from forebrain and brainstem structures (Geisler et al. 2007).

Self-administration and abuse of drugs, like ICSS, entails the copious expenditure of behavioral resources in pursuit of an objective that appears worthless or detrimental to biological fitness. A full account of the neural circuitry responsible for ICSS is likely to provide insight into how dependence-inducing drugs attain their powerful influence over behavior and decision making. Performance for BSR is enhanced by many drugs that are both self-administered by 
laboratory animals and subject to abuse by humans (Wise 1996). The dominant hypothesis concerning these effects is that the drugs in question alter dopaminergic neurotransmission directly or indirectly. Much recent work on ICSS and reward seeking is directed at inputs to ventral tegmental dopamine neurons or to their postsynaptic targets, which must be considered as possible substrates of drug action in addition to the dopamine neurons themselves.

\section{Schools of Thought}

Theoretical accounts of ICSS have emphasized different aspects of the phenomenon. However, the differences are often less marked than the contrasting labels for the theories suggest.

Hedonic. An early article on ICSS in Scientific American bears the provocative title: "Pleasure Centers in the Brain" (Olds 1956). When someone first observes a self-stimulating rat, they typically speculate that the stimulation "makes the rat feel good." It seems commonsensical that one would tend to repeat an activity that produced pleasurable consequences. The converse, however, is far from clear. Does the fact that an activity is repeated necessarily imply that it generates a positive hedonic state? Will the activity be repeated if, and only if, it is pleasurable?

Pleasure implies awareness, a faculty believed to depend on resources in short supply, such as attention and working memory. It would be highly maladaptive to pass all signals controlling action through such a narrow processing bottleneck. Thus, contemporary depictions of the control of action place most of the burden on distributed processes operating below the waterline of awareness (Baars 1988; LeDoux 1996). On that view, the repeated pressing of the lever does not necessarily imply a positive hedonic state (Shizgal 1999).

Theorists who have ventured onto this slippery terrain have typically been more circumspect than the labels for their theories might suggest (Wise 1982; Wise 2008; Kringelbach \& Berridge 2009). Their task is not easy. On the one hand, they cannot ignore reports from early studies of human patients who voluntarily self-administered electrical stimulation to sites homologous to those that support ICSS in laboratory animals. These accounts include descriptions of pleasurable experience (Sem-Jacobsen 1959; Heath 1964). On the other hand, some have contested the interpretation of the verbal subjective reports from these studies of human patients (Kringelbach \& Berridge 2009), and we lack the means to infer the subjective experience of nonhuman animals. Moreover, subjective pleasure and persistent operant responding for rewards have been dissociated in studies of human participants (Lamb et al. 1991).

Seeking and craving. If a path to the lever is blocked by an obstacle, the self-stimulating rat will seek another. Such flexible approach to a goal is typical of what psychologists call appetitive behavior, and it contrasts with the more stereotyped patterns observed once the animal procures a natural goal object, such as food, and begins to consume it. Potentiation of flexible approach figures heavily in several different accounts of the ICSS phenomenon. Although Wise applied the psychiatric term, anhedonia, to his theory of why reductions in dopaminergic neurotransmission attenuate ICSS, drug self-administration, and operant performance for natural rewards, the core of his formulation concerns what he calls "motivational arousal," a state that promotes flexible reward seeking. His theory differs from several others in that he views subjective pleasure as a 
normal, but not obligatory, concomitant of the motivational arousal engendered by exposure to rewards. In contrast, the incentive-sensitization hypothesis of Robinson and Berridge (1993; 1998) portrays the ICSS phenomenon as a manifestation of what they call 'wanting,' a process that endows stimuli with the ability to seize attention and to promote anticipatory, flexible approach (Ikemoto \& Panksepp 1999). They argue that dopaminergic neurotransmission is essential to 'wanting' but not to a contrasting component of reward that they term 'liking.' When 'liking' impinges on consciousness, it is experienced as pleasure. It is also manifested in consummatory behavior so that we ingest foods that we like and eschew foods that we do not. The subjective states of rats are hidden from us, but their ingestive behavior is not. Thus, Berridge and his co-workers use 'liking' in quotation marks to describe behavior that they believe to arise from the neural underpinning of hedonic states, whether these states are experienced or not. Experimenter-delivered stimulation of ICSS sites fails to strengthen ingestive patterns and may weaken them, a finding that these theorists take to reflect a dissociation between 'wanting' and 'liking.' Although this interpretation has been contested (Wise 2008), the distinction between wanting and liking has been highly influential, particularly because of its correspondence to the self-reports of long-time drug users who evince continued cravings but claim that the drug no longer generates euphoric effects. Indeed, in studies of human participants, partial blockade of dopamine synthesis reduced drug craving without systematically undermining self-reported euphoric effects (Leyton et al. 2007).

Reinforcement learning. Self-stimulation of many brain sites is acquired quickly, and the behavior typically extinguishes rapidly once the reward is no longer delivered. This rapid reinforcement learning is among the most striking aspects of the ICSS phenomenon, and it has played a prominent role in theoretical accounts. During the 1990s, the confluence of work in machine learning and electrophysiology cast the reinforcement-learning aspects of ICSS in a new light.

In their quest to develop intelligent machines, researchers came to appreciate that much of the knowledge required to solve real-world problems cannot be specified in advance. The environment is not entirely predictable, and its complexity is effectively without limit. Under such conditions, it is desirable to imbue machines with the ability to learn about the consequences of their actions and the relationships between events. The algorithms developed to meet these objectives share important properties with formulations developed by students of animal learning while extending the quantitative rigor and scope of those ideas (Sutton \& Barto 1998). One such algorithm, called temporal-difference learning, entails moment-to-moment updating of predictions concerning future rewards and comparison of these predictions to the rewards actually received. Discrepancies between predictions and outcomes, called temporaldifference errors, cause reward predictions and the associated reward-seeking actions to be modified and improved.

Montague, Dayan and Sejnowski recognized a striking correspondence between the properties of temporal difference errors and the activity of midbrain dopamine neurons observed by Schultz and colleagues during Pavlovian and operant conditioning experiments carried out in monkeys (Montague et al. 1996; Schultz et al. 1997). They proposed that this correspondence could account for the ICSS phenomenon. According to this view, the brief burst of ("phasic") 
firing in midbrain dopamine neurons that is triggered by electrical stimulation constitutes a temporal-difference error that alters weights in the neural networks encoding the value of the reward-seeking actions and the predictions associated with response-related cues. In effect, the self-stimulating rat is portrayed as the victim of a hallucination of value. Although the stimulation produces no homeostatic or reproductive benefit, cues that predict it and the actions that procure it come to be represented as increasingly valuable due to the reward prediction errors it generates.

A problem with the original formulation of the prediction-error hypothesis of ICSS is that the weights increase progressively with delivery of successive stimulation trains and should saturate eventually regardless of the strength of any suprathreshold stimulation train; saturation would be achieved rapidly in the case of strong trains and more slowly in the case of weaker ones. This prediction doesn't jibe well with the finding that rats systematically choose higher strength trains in preference to weaker ones, even after extensive experience with the stimulation (Simmons \& Gallistel 1994). The evidence that midbrain dopamine neurons are activated transsynaptically by MFB stimulation (Shizgal 1997) can rescue the hypothesis: an inhibitory input representing the reward prediction could come to counteract the transient activation produced by the stimulation train, reducing and eventually eliminating the prediction error. Such a nulling effect should be much less likely in the case of optical stimulation, which can excite the dopamine neurons directly. Substituting optical for electrical stimulation could provide a powerful test of the hypothesis that induced bursts of firing in dopamine neurons are the cause of the observed reward-related learning.

Neuroeconomic. The study of the neural basis of decision making and the evaluation of the benefits, costs, and risks on which choices are based has come to be called neuroeconomics (Glimcher \& Rustichini 2004; Loewenstein et al. 2008). The first papers written from this perspective were accounts of ICSS (Shizgal \& Conover 1996; Shizgal 1997), and it has been argued that there is a striking correspondence between the neuroeconomic approach to ICSS and some core ideas in economics, judgment, and decision making (Shizgal 2012). The neuroeconomic approach shares with the reinforcement-learning approach a commitment to quantitative models that can be tested by means of behavioral and neural measurements. One distinction between the approaches concerns the phase of the experiment that is of primary interest. The reinforcement-learning approach focuses primarily on how relationships between events and contingencies between actions and their consequences are learned. In contrast, neuroeconomic experiments typically begin with a subject that has already learned a task. In the case of ICSS, such work has focused a) on the psychophysical transformations that translate objective variables, such as the strength and cost of rewards, into their subjective equivalents, and $b$ ) on the stages of processing at which particular neural populations contribute to the computation of reward value (Hernandez et al. 2010). That said, the reinforcement-learning approach has been extended to address the issue of reward-related costs; whereas this approach links reward-related learning to phasic firing of dopamine neurons, effort costs have been related to more slowly varying, tonic levels of dopamine (Niv et al. 2007). 


\section{Methodological Issues and Emerging Directions}

Biophysical specificity. Studies employing electrical stimulation of the central nervous system have been plagued by a lack of biophysical specificity. The stimulation typically activates a set of neurons of unknown composition in addition to the population targeted by the experimenter. This is the principal reason why the directly stimulated neurons that give rise to the rewarding effect have proved so difficult to identify. The recent development of optogenetic methods promises to circumvent the lack of specificity inherent in electrical stimulation methods while retaining all of the principal advantages (Deisseroth 2010; Yizhar et al. 2011).

Using optogenetic methods, neural activity can be silenced or induced on timescales as short as that corresponding to single nerve impulses and as long as the actions of many drugs. These effects can be restricted to specific neural populations targeted on the basis of conditional gene expression, cell-body location, and axonal projections. The optical component of optogenetic technologies consists of two elements: molecules that transduce light into cellular signals and a mechanism to deliver appropriate photic stimuli to the targeted cells. The transducer molecules are opsins, the class of proteins that render photoreceptors sensitive to light. Unlike vertebrate opsins, which compose part of a multi-stage chain of complex chemical reactions, microbial opsins are far simpler, functioning directly as light-gated ion channels or pumps that alter the movement of electrical charge across the cell membrane so as to either promote or suppress the generation of neural signals. The genetic component of optogenetic technologies consists of methods for inserting microbial opsin genes into the genome of neurons, restricting the expression of these genes to specifically targeted cell populations and promoting trafficking of the protein products of these genes to the cell membrane. Fine fiber-optic probes implanted in the brain are used to deliver light at a wavelength selected for optimal capture by the microbial opsin. Optogenetic methods are complemented by molecular neuroanatomical techniques for labeling and visualizing specific populations of neurons.

The initial attempts to train rodents to perform operant responses to obtain optical stimulation of ventral tegmental dopamine neurons were only moderately successful. In one case (Adamantidis et al. 2011), mice could be trained to lever press only if food was delivered along with the laser illumination; however, operant performance was subsequently maintained by optical stimulation alone, albeit at low response rates. In a second study (Kim et al. 2012), mice were successfully trained to nose poke for optical stimulation of ventral tegmental neurons but again, response output was low. In contrast, rats expressing channelrhodopsin2, an excitatory opsin, in dopamine neurons respond in a vigorous, sustained manner for optical stimulation of the ventral tegmental area (Witten et al. 2011). This work and associated studies in which specific inputs to ventral tegmental dopamine neurons were manipulated optogenetically (Britt et al. 2012; Jennings et al. 2013) demonstrate the promise of the new methods to overcome technical obstacles to determining the neural underpinnings of reward seeking.

Behavioral specificity. Another challenge to achieving an adequate neurobehavioral account of ICSS and of reward seeking in general arises from what can be called "multistage causal convergence." There are many possible reasons why a laboratory animal may choose to perform an operant response. The various processes that can support such responses are likely composed 
of multiple stages. For example, the decision to press a lever may arise from the separate computation of benefits, costs, and risks, the folding together of the results of these computations into a unitary estimate of value that is compared with estimates of the values of other available actions, and the translation of the winning estimate into reward-seeking actions. When the proclivity of the animal to press the lever is altered by a neural manipulation, how is one to know which of the possible causes of lever pressing has been affected and at what stage of processing? Answering such questions requires that the specificity and power of behavioral measurement methods match those of the promising new methods for optical stimulation, optical silencing, and neuroanatomical visualization.

Work employing a neuroeconomic approach provides an example of the increasing specificity of new behavioral methods and the multistage nature of the processes underlying ICSS. It has long been known that psychomotor stimulants boost performance for BSR, an effect that been attributed to a drug-induced increase in the sensitivity of reward-related circuitry (Crow 1970). This hypothesis was tested by means of a multistage model of ICSS coupled to a behavioral testing paradigm that can distinguish the signatures of drug action at different stages of processing (Hernandez et al. 2010). According to this model, the effect of the volley of action potentials triggered by the rewarding stimulation is integrated according to an aggregate rate code; reward strength is represented by the number of firings within a time window, integrated across neurons. This count is transformed non-linearly into a subjective representation of reward intensity (Simmons \& Gallistel 1994) and then combined with information about reward cost and probability so as to yield an estimate of the payoff from lever pressing. If psychomotor stimulants increase the sensitivity of the circuitry to electrical activation, these drugs should lower the number of firings required to produce a reward of given intensity. By measuring ICSS as a function of both the strength and cost of rewarding stimulation, Hernandez and colleagues showed that cocaine enhances reward seeking primarily by other means, such as a reduction in subjective reward cost or amplification of the output of the circuitry performing the integration (Hernandez et al. 2010).

Emerging directions. Optogenetic experiments on reward seeking are typically complemented by electrophysiological measurement of neural activity. Integration of these approaches with increasingly specific behavioral testing methods and with quantitative modeling in the reinforcement-learning, psychophysical and neuroeconomic traditions may address many of the problems that have hindered progress in the study of ICSS and of the neural basis of reward seeking. This would provide a solid foundation for testing competing accounts of how goals are evaluated, selected, and sought, and how information about benefits, costs, and risks is learned, used, and abused.

\section{Keywords}

reward, reinforcement, motivation, learning, pleasure, hedonic, dopamine, positive psychology, addiction, optogenetics 


\section{Acknowledgements}

The author's research on brain stimulation reward has been supported by the Canadian Institutes of Health Research, the Natural Science and Engineering Research Council of Canada, the Fonds de Recherche du Québec (Santé), and the Concordia University Research Chairs program. Brian Dunn, Daniel Palacios, and Sarah Nolan-Poupart provided helpful comments on the manuscript.

\section{References}

Adamantidis, A.R. et al., 2011. Optogenetic Interrogation of Dopaminergic Modulation of the Multiple Phases of Reward-Seeking Behavior. The Journal of neuroscience : the official journal of the Society for Neuroscience, 31(30), pp.10829-10835.

Baars, B.J., 1988. A cognitive theory of consciousness, Cambridge England ; New York: Cambridge University Press.

Berridge, K.C. \& Robinson, T.E., 1998. What is the role of dopamine in reward: hedonic impact, reward learning, or incentive salience? Brain research Brain research reviews, 28(3), pp. 309-369.

Blundell, J.E. \& Herberg, L.J., 1968. Relative effects of nutritional deficit and deprivation period on rate of electrical self-stimulation of lateral hypothalamus. Nature, 219(5154), pp.627628.

Britt, J.P. et al., 2012. Synaptic and Behavioral Profile of Multiple Glutamatergic Inputs to the Nucleus Accumbens. Neuron, 76(4), pp.790-803.

Conover, K.L. \& Shizgal, P., 1994a. Competition and summation between rewarding effects of sucrose and lateral hypothalamic stimulation in the rat. Behavioral Neuroscience, 108(3), pp.537-548.

Conover, K.L. \& Shizgal, P., 1994b. Differential effects of postingestive feedback on the reward value of sucrose and lateral hypothalamic stimulation in rats. Behavioral Neuroscience, 108(3), pp.559-572.

Conover, K.L., Woodside, B. \& Shizgal, P., 1994. Effects of sodium depletion on competition and summation between rewarding effects of salt and lateral hypothalamic stimulation in the rat. Behavioral Neuroscience, 108(3), pp.549-558. 
Crow, T.J., 1970. Enhancement by cocaine of intra-cranial self-stimulation in the rat. Life sciences, 9(7), pp.375-381.

Deisseroth, K., 2010. Controlling the brain with light. Scientific American, 303(5), pp.48-55.

Delgado, J.M., Roberts, W.W. \& Miller, N.E., 1954. Learning motivated by electrical stimulation of the brain. The American journal of physiology, 179(3), pp.587-593.

Edmonds, D.E. \& Gallistel, C.R., 1974. Parametric analysis of brain stimulation reward in the rat: III. Effect of performance variables on the reward summation function. Journal of Comparative and Physiological Psychology, 87(5), pp.876-883.

Forster, G.L. et al., 2002. M5 muscarinic receptors are required for prolonged accumbal dopamine release after electrical stimulation of the pons in mice. Journal of Neuroscience, 22(1), p.RC190.

Fulton, S., Woodside, B. \& Shizgal, P., 2000. Modulation of brain reward circuitry by leptin. Science (New York, NY), 287(5450), pp.125-128.

Gallistel, C.R. \& Beagley, G., 1971. Specificity of brain stimulation reward in the rat. Journal of Comparative and Physiological Psychology, 76(2), pp.199-205.

Geisler, S. et al., 2007. Glutamatergic afferents of the ventral tegmental area in the rat. The Journal of neuroscience : the official journal of the Society for Neuroscience, 27(21), pp. $5730-5743$.

Glimcher, P.W. \& Rustichini, A., 2004. Neuroeconomics: the consilience of brain and decision. Science (New York, NY), 306(5695), pp.447-452.

Green, L. \& Rachlin, H., 1991. Economic substitutability of electrical brain stimulation, food, and water. Journal of the Experimental Analysis of Behavior, 55(2), pp.133-143.

Heath, R.G., 1964. Pleasure response of human subjects to direct stimulation of the brain: physiologic and psychodynamic considerations. In R. G. Heath, ed. The role of pleasure in behavior. New York: Harper \& Row, pp. 219-243.

Hernandez, G. et al., 2010. At what stage of neural processing does cocaine act to boost pursuit of rewards? PLoS ONE, 5(11).

Hess, W., 1949. The Central Control of the Activity of Internal Organs. Nobelprize.org. Available at: http://www.nobelprize.org/nobel_prizes/medicine/laureates/1949/hess-lecture.html [Accessed March 10, 2013].

Hoebel, B.G. \& Teitelbaum, P., 1962. Hypothalamic control of feeding and self-stimulation. Science (New York, NY), 135(3501), pp.375-377. 
Ikemoto, S. \& Panksepp, J., 1999. The role of nucleus accumbens dopamine in motivated behavior: a unifying interpretation with special reference to reward-seeking. Brain research Brain research reviews, 31(1), pp.6-41.

Jennings, J.H. et al., 2013. Distinct extended amygdala circuits for divergent motivational states. Nature.

Kim, K.M. et al., 2012. Optogenetic mimicry of the transient activation of dopamine neurons by natural reward is sufficient for operant reinforcement. PLOS ONE, 7(4), p.e33612.

Kringelbach, M.L. \& Berridge, K.C., 2009. Towards a functional neuroanatomy of pleasure and happiness. Trends in Cognitive Sciences, 13(11), pp.479-487.

Lamb, R.J. et al., 1991. The reinforcing and subjective effects of morphine in post-addicts: a dose-response study. The Journal of pharmacology and experimental therapeutics, 259(3), pp.1165-1173.

LeDoux, J.E., 1996. The emotional brain : the mysterious underpinnings of emotional life, New York: Simon \& Schuster.

Leyton, M. et al., 2007. Mood-elevating effects of d-amphetamine and incentive salience: the effect of acute dopamine precursor depletion. Journal of psychiatry \& neuroscience : $J P N$, 32(2), pp.129-136.

Loewenstein, G., Rick, S. \& Cohen, J., 2008. Neuroeconomics. Annual Review of Psychology, 59, pp.647-672.

Margules, D.L. \& Olds, J., 1962. Identical "feeding" and "rewarding" systems in the lateral hypothalamus of rats. Science (New York, NY), 135, pp.374-375.

Miguelez, M. \& Bielajew, C., 2004. Mapping the neural substrate underlying brain stimulation reward with the behavioral adaptation of double-pulse methods. Reviews in the neurosciences, 15(1), pp.47-74.

Milner, P.M., 1989. The discovery of self-stimulation and other stories. Neuroscience \& Biobehavioral Reviews, 13(2-3), pp.61-67.

Moisan, J. \& Rompré, P.P., 1998. Electrophysiological evidence that a subset of midbrain dopamine neurons integrate the reward signal induced by electrical stimulation of the posterior mesencephalon. Brain research, 786(1-2), pp.143-152.

Montague, P.R., Dayan, P. \& Sejnowski, T.J., 1996. A framework for mesencephalic dopamine systems based on predictive Hebbian learning. The Journal of neuroscience : the official journal of the Society for Neuroscience, 16(5), pp.1936-1947. 
Moruzzi, G. \& Magoun, H.W., 1949. Brain stem reticular formation and activation of the EEG. Electroencephalography and Clinical Neurophysiology, 1(1-4), pp.455-473.

Niv, Y. et al., 2007. Tonic dopamine: opportunity costs and the control of response vigor. Psychopharmacology, 191(3), pp.507-520.

Olds, J., 1973. Commentary on Olds, J. and Milner, P. Positive reinforcement produced by electrical stimulation of septal area and other regions of rat brain. In E. S. Valenstein, ed. Brain stimulation and motivation; research and commentary. Glenview, Ill.,: Scott Foresman and Company, pp. 81-99.

Olds, J., 1956. Pleasure Centers in the Brain. Scientific American, 195(4), pp.105-117.

Olds, J., 1958a. Satiation effects in self-stimulation of the brain. Journal of Comparative and Physiological Psychology, 51(6), pp.675-678.

Olds, J., 1958b. Self-stimulation of the brain; its use to study local effects of hunger, sex, and drugs. Science (New York, NY), 127(3294), pp.315-324.

Olds, J. \& Milner, P., 1954. Positive reinforcement produced by electrical stimulation of septal area and other regions of rat brain. Journal of Comparative and Physiological Psychology, 47(6), pp.419-427.

Rada, P.V. et al., 2000. Acetylcholine release in ventral tegmental area by hypothalamic selfstimulation, eating, and drinking. Pharmacology, biochemistry, and behavior, 65(3), pp. $375-379$.

Robinson, T.E. \& Berridge, K.C., 1993. The neural basis of drug craving: an incentivesensitization theory of addiction. Brain research Brain research reviews, 18(3), pp.247291.

Routtenberg, A. \& Lindy, J., 1965. Effects of the availability of rewarding septal and hypothalamic stimulation on bar pressing for food under conditions of deprivation. Journal of Comparative and Physiological Psychology, 60(2), pp.158-161.

Schultz, W., Dayan, P. \& Montague, P.R., 1997. A neural substrate of prediction and reward. Science (New York, NY), 275(5306), pp.1593-1599.

Sem-Jacobsen, C.W., 1959. Depth-electrographic observations in psychotic patients: a system related to emotion and behavior. Acta psychiatrica Scandinavica. Supplementum, 34(136), pp.412-416.

Shizgal, P., 1997. Neural basis of utility estimation. Current Opinion in Neurobiology, 7(2), pp. 198-208. 
Shizgal, P., 1999. On the neural computation of utility: implications from studies of brain stimulation reward. In D. Kahneman, E. Diener, \& N. Schwarz, eds. Well Being: Foundations of Hedonic Psychology. New York: Russell Sage Foundation, pp. 502-526.

Shizgal, P., 2012. Scarce means with alternative uses: Robbins' definition of economics and its extension to the behavioral and neurobiological study of animal decision making. Frontiers in Neuroscience, 6, p.20.

Shizgal, P. \& Conover, K., 1996. On the neural computation of utility. Current Directions in Psychological Science, 5(2), pp.37-43.

Shizgal, P. \& Murray, B., 1989. Neuronal basis of intracranial self-stimulation. In J. M. Liebman \& S. J. Cooper, eds. The neuropharmacological basis of reward. Oxford: Oxford University Press, pp. 106-163.

Simmons, J.M. \& Gallistel, C.R., 1994. Saturation of subjective reward magnitude as a function of current and pulse frequency. Behavioral Neuroscience, 108(1), pp.151-160.

Stellar, E., 1954. The physiology of motivation. Psychological Review, 61(1), pp.5-22.

Stuber, G.D. et al., 2011. Excitatory transmission from the amygdala to nucleus accumbens facilitates reward seeking. Nature.

Sutton, R.S. \& Barto, A.G., 1998. Reinforcement learning : an introduction 1st ed, Cambridge, Mass.: MIT Press.

Valenstein, E.S., 1973. History of brain stimulation: investigations into the physiology of motivation. In E. S. Valenstein, ed. Brain stimulation and motivation; research and commentary. Glenview, Ill.,: Scott Foresman and Company.

Wise, R.A., 1996. Addictive drugs and brain stimulation reward. Annual review of neuroscience, 19, pp.319-340.

Wise, R.A., 2008. Dopamine and reward: the anhedonia hypothesis 30 years on. Neurotoxicity research, 14(2-3), pp.169-183.

Wise, R.A., 1982. Neuroleptics and operant behavior: The anhedonia hypothesis. The Behavioral and brain sciences, 5, pp.39-97.

Wise, R.A. \& Rompré, P.P., 1989. Brain dopamine and reward. Annual Review of Psychology, 40, pp.191-225.

Witten, I.B. et al., 2011. Recombinase-Driver Rat Lines: Tools, Techniques, and Optogenetic Application to Dopamine-Mediated Reinforcement. Neuron, 72(5), pp.721-733. 
Yeomans, J.S. et al., 2000. Brain-stimulation reward thresholds raised by an antisense oligonucleotide for the M5 muscarinic receptor infused near dopamine cells. The Journal of neuroscience : the official journal of the Society for Neuroscience, 20(23), pp.88618867.

Yizhar, O. et al., 2011. Optogenetics in neural systems. Neuron, 71(1), pp.9-34. 